\section{Einheitliche Definition von Exazerbationen bei Bronchiektasen}

Hill AT et al. Pulmonary exacerbation in adults with bronchiectasie: a consensus definition for clinical research. Eur Respir J 2017; 49: doi:10.1183/13993003.00051-2017

In vielen Studien, in denen Therapien für Erwachsene mit Bronchiektasen untersucht werden, sind die Kriterien Exazerbationshäufigkeit und Zeit bis zur ersten Exazerbation entscheidende Endpunkte. Die verwendeten Definitionen der Exazerbation sind allerdings heterogen, was die Vergleichbarkeit der Therapieeffekte einschränkt. Die jetzt veröffentlichte, konsentierte Definition kann für künftige Studien genutzt werden.

Grundlage der Definitionsentwicklung war eine systematische Auswertung der zwischen den Jahren 2000 und 2015 in klinischen Studien verwendeten Exazerbationsdefinitionen bei erwachsenen Patienten mit Bronchiektasen ohne Verbindung zu zystischer Fibrose. An dem Prozess waren Bronchiektasie-Experten aus Europa (European Multicentre Bronchiectasis Research Collaboration, EMBARC), Nordamerika (US Brochiectasis Research Registry/COPD Foundation, BRR), Afrika und Australien beteiligt. Die Konsensbildung erfolgte im Delphi-Verfahren. Für die Konsensusdefinition der Exazerbation wurden Kriterien berück- sichtigt, wenn sie von $\geq 80 \%$ der Experten als sehr starker Hinweis auf eine Exazerbation oder die Kriterien für die Definition einer Exazerbation als unerlässlich betrachtet wurden.

\section{Konsentierte Definition}

Insgesamt identifizierte die Arbeitsgruppe 50 Veröffentlichungen mit 20 verschiedenen Exazerbationsdefinitionen. Mehr als $80 \%$ der publizierten Kriterien schlossen die Notwendigkeit einer Antibiotikagabe ein sowie, jeweils zunehmend, Dyspnoe, Husten, Sputumvolumen, Veränderungen der Sputumfarbe. Am Ende des Delphi-Verfahrens gingen folgende Symptome in die Definition ein: Hämoptysen, Brustschmerz, abnehmende körperliche Belastbarkeit, Giemen, Hypoxie, Fieber, Auskultationsbefunde, von Patienten berichtete Exazerbationen, systemische Störung, Fatigue, Zunahme von Kurzatmigkeit $\geq 24$ h, von Husten und von Sputumvolumen, veränderte Sputumfarbe, Antibiotikagabe. Entsprechend des gebildeten Konsensus lautet die aktuelle Definition einer Exazerbation bei Bronchiektasen: Verschlechterung von mindestens 3 entscheidenden Symptomen (Husten, Sputumvolumen und/oder -konsistenz, Sputumpurulenz, Atemnot und/oder körperliche Belastbarkeit, Fatigue und/oder allgemeines Krankheitsgefühl, Hämoptyse) und die ärztliche Entscheidung zur Therapieänderung.
FAZIT

Mit dieser Konsensdefinition der Exazerbation soll es nach Ansicht der Autoren möglich sein, Ergebnisse klinischer Studien mit erwachsenen Bronchiektasenpatienten zu standardisieren und die Therapieeffekte verschiedener Interventionen aussagekräftiger vergleichen zu können.

Matthias Manych, Berlin 